PAPER 76

\title{
A SUMMARY AND ASSESSMENT OF CURRENT 2I-CM RESULTS CONCERNING SPIRAL AND DISK STRUCTURES IN OUR GALAXY
}

\author{
J. H. OORT
}

Leiden Observatory, The Netherlands

The results of the survey of the galactic system in the hydrogen line made in the Netherlands have been extensively published and have been discussed at the symposium in Manchester and the I.A.U. assembly in Dublin. I shall not enter into a renewed discussion of these, but rather consider what has been added since.

The results referred to had two serious limitations: in the first place they were restricted to the part of the Milky Way that rose above the Dutch horizon; in the second place they were not sufficiently refined to reveal the true structure of the central region of our Galaxy.

Both limitations have now been largely removed. The observations by Kerr and his collaborators have yielded the structure of the other part of the Milky Way; while refined observations with the 25-meter radio telescope at Dwingeloo have given considerably more insight into what takes place in the Galaxy's central regions.

I shall briefly consider the structure of the whole as it now appears from the combined Sydney and Leiden measures:

1. We comment first on the thinness of the layer. The distance between half-density points is about 220 parsecs, and is much the same in all parts of the system, except in the central part.

2 . In the second place we consider the remarkable flatness of the layer. Within $R=10$ the points of maximum density in columns perpendicular to the galactic plane are nowhere more than 75 parsecs from the mean plane, except in small areas near $R=10$. Within $R=6$ kiloparsecs the deviations are even smaller (all $<30$ parsecs). Further studies of this remarkable phenomenon may eventually give us a better insight into how the galactic system has evolved. It is also interesting that we can clearly observe the limits of $R$ to which this evolution has been completed. In the low-density regions outside $R=12$, deviations running up to 600 parsecs are observed. These deviations have a clearly systematic character.

The flatness for not too large $R$ offers a basis for determining the galactic pole with an accuracy that is an order of magnitude better than what could be attained previously.

* For a more extensive summary and for illustrations the reader is referred to the article "The Galactic System as a Spiral Nebula" [1]. 
3. The $21-\mathrm{cm}$ measures provide us with valuable data on the velocity of rotation for $R<8$ kiloparsecs. The data from opposite sides of the center are satisfactorily concordant. The part within $R=2.5$ kiloparsecs is quite uncertain because of the large deviations from circular motion in the central region, which will be discussed below.

From the observations by Guido Münch in particular, a number of valuable data are available from distant $O B$ stars. These data, which are more direct because distances are also available, fit in well with the $21-\mathrm{cm}$ data (unpublished).

4. The derivation of the hydrogen distribution in the plane is based on this rotation curve. The principal uncertainties are in the absolute scale (the uncertainty in the distance $R_{0}$ of the sun from the center may be estimated at about 10 per cent), in the run of the rotation curve outside $R=R_{0}$, in the systematic deviations from circular motions, and in the corrections for random motions. The last two are the most serious; the picture one obtains depends considerably on the amount of random motion that is supposed to exist. The correction for this has a great influence on the extent to which the hydrogen is found to be concentrated in the arms. Our knowledge of the random motions in different regions is still insufficient.

Comparison of the northern and southern observations shows that there is very good agreement in the large overlapping region. In Fig. 1 the part between longitudes 340 and 210 degrees is based on the Leiden observations, while the sector between 210 and 312 degrees is derived from the Sydney measurements. The contours show the densities in the layer of maximum density (in some of the outer regions this layer deviates from the mean galactic plane). The difference in general appearance of the northern and southern regions is partly the result of slightly different corrections for random motions, and also of the manner in which the line profiles were deduced.

There are evidently some quite long stretches of dense parts through which we can trace the spiral arms. But the combination with the southern observations is disappointing in so far as it does not yet permit us to outline unambiguously a simple spiral pattern traceable through the whole Galaxy. What makes this so difficult is the combination of the irregularity which is characteristic of all spiral galaxies and the existence of the large gap in our picture between $l=315$ degrees and $l=340$ degrees. The indirect way in which the distribution of the gas was derived has also added considerably to the untidiness of the picture.

We can, nevertheless, get some idea of the spacing of the principal arms, which may be estimated to be between 2 and $2 \frac{1}{2}$ kiloparsecs apart. If there are two principal arms this would correspond to an inclination of roughly 5 degrees.

5. An interesting datum found from the observations is the way in which the over-all density in the galactic plane varies with $R$. The density shows a maximum at $R=6.5$ kiloparsecs where it is about 1 atom $/ \mathrm{cm}^{3}$. It diminishes to less than 0.1 atom $/ \mathrm{cm}^{3}$ at about $R=14$ kiloparsecs. Westerhout has recently derived the density distribution of ionized hydrogen. This reaches 


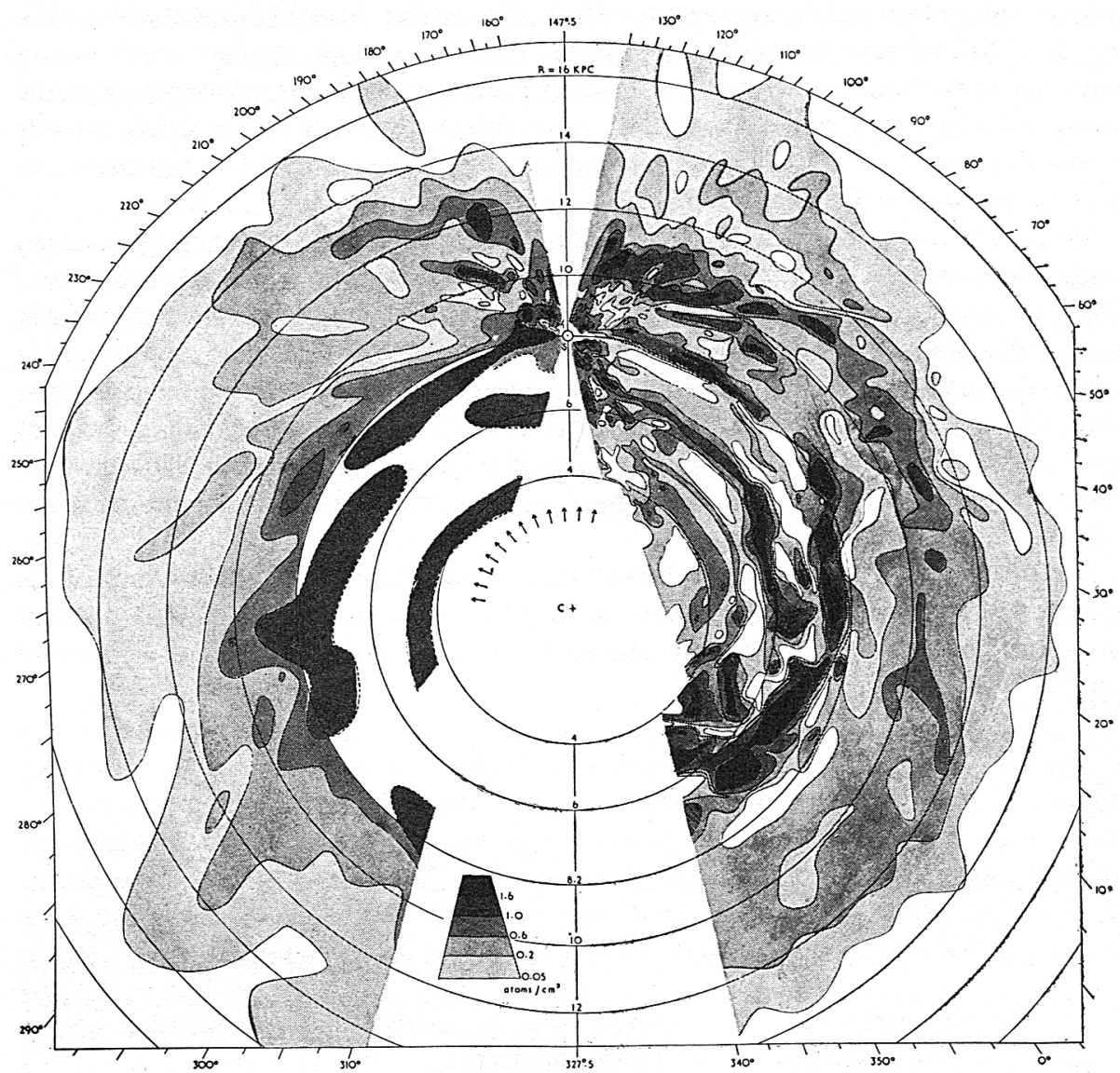

FIG. 1. Distribution of neutral hydrogen in the galactic system.

a much steeper maximum around $R=4$, with a density of roughly 0.5 . Except in the ring between 3 and $4 \frac{1}{2}$ kiloparsecs the ionized-hydrogen density is much less than the neutral-gas density. Everywhere the density of the gas is only a small fraction of the total mass density, except in the outermost regions of the galactic disk, beyond $R=10$ kiloparsecs.

6. The study of the hydrogen in the region within 3 kiloparsecs from the center has revealed quite unexpected phenomena. Westerhout first observed them in 1954 in the course of determining the galactic rotation as a function of the distance from the center. In the beginning of 1957 van Woerden and Rougoor found that the strong arm observed near the region's outer border had an outward motion of $53 \mathrm{~km} / \mathrm{second}$ superimposed upon its motion around the center. The outward motion was measured at the point where the arm crosses the line joining the sun and the galactic center. At this point the arm is seen in absorption against Sagittarius A. Other features in the central 
region were observed to move away from the center at still higher velocities, up to $130 \mathrm{~km} / \mathrm{second}$. They seem, at the same time, to take part in the rotation of the galactic system. Detailed measures by Rougoor (paper 77) have indicated that very probably all the gas in the region within $R=3$ kiloparsecs is moving outward. Beside much irregularity there is also the indication of a large-scale structure, presumably of spiral character.

Although the observations clearly show high outward velocities as well as high rotation velocities, it is not possible to obtain a clear separation between the two components in individual regions. We do not yet know, therefore, how the systematic outward velocity varies with the distance from the center. The absence of long low-intensity wings in line profiles for directions that are more than about 22 degrees from the center has led us to believe that the abnormal motions are mainly restricted to a region within 3 kiloparsecs from the center, but it is not impossible that smaller expansional motions persist beyond this distance.

Because of our incomplete knowledge about the systematic motions it is impossible at present to determine the manner in which the average density of neutral hydrogen varies with the distance from the center in this nuclear region.

7. The existence of a source of continuous radio-frequency radiation in a "corona" around the galactic system indicates the presence of considerable quantities of gas at large distances from the galactic plane. Part of this may be in the form of neutral clouds. So far, no adequate observations of neutral gas in the "corona" have been made. The intensities must certainly be very low, but in view of the stability of the zero line now obtained by Muller, a successful attack may become possible in the near future.

The 21-cm measures have contributed in several ways toward a better insight into the phenomenon of spiral structure.

In the first place they have outlined this structure in a system for which we have considerable knowledge of differential rotation. Assuming that the arms in the galactic system, when considered on a very large scale, form a more or less continuous pattern through the entire system, as in M 51, we conclude from the data that without a permanent rebuilding process the arms would dissolve in a period of between 100 and 200 million years. Similar conclusions can also be drawn from M 31 and $M 81$; in the latter case the rotation has been found from optical observations.

The rebuilding of the arms may be effected by the internal random motions in the interstellar medium. The $21-\mathrm{cm}$ measures are capable of furnishing a great deal of information about these motions. Furthermore, it may be hoped that ultimately the study of the distribution of star clusters of different ages and their systematic deviation from the gas arms may indicate the nature of the process by which the spiral arms are maintained against the stretching effect of the differential rotation.

The extreme flatness of the layer indicates probably that there has been a considerable exchange of material from the inner to the outer parts. 
It is plausible to think that the large motions away from the center in the part with a 3-kiloparsec radius have some connection with the formation of the spiral pattern in this region. These phenomena may ultimately give us a clue to the spiral structure in general. It seems already evident from the observed motions that magnetic fields must play a dominant role in causing the expansion and in imparting the necessary angular momentum to the expanding gas.

It is clear that at the present moment we are still quite far from understanding the origin and the maintenance of spiral structure. But the great extension of observable data, which has become possible by radio-astronomical methods, offers at least some hope that important progress will be made in the coming years.

\section{REFERENCE}

[1] Oort, J. H., Kerr, F. J., and Westerhout, G. M.N.R.A.S. 118, 379, 1958.

\section{Discussion}

Bok: We rightfully pay most attention now to the regions of the galactic center. In $21-\mathrm{cm}$ research we should, however, continue to pay careful attention to the sections of the Milky Way at distances from the center greater than that of the sun. We should urge our optical colleagues to measure radial velocities and estimate distances of remote objects in the anti-center half of the Milky Way. The optical astronomer is not excessively bothered by interstellar absorption in the anti-center section. It is a straightforward matter to determine the curve for the variation of rotational velocity with distance from the galactic center for OB stars. Once this curve is adequately known we can derive from it important dynamical properties of our Galaxy. It also finds immediate application in the interpretation of $21-\mathrm{cm}$ profiles.

Oort: I am in the fullest agreement with what Dr. Bok has said about the importance of more optical data in the outer parts. I have on several occasions especially emphasized this. Optical observations are our only hope of determining deviations from circular motion.

Becker: Is there any good reason to assume that the planes of symmetry of the stars and of the gas are identical?

Oort: Even if one admit the possibility that the gravitational plane of symmetry may not be identical with that of the gas, the fact alone that the gas is confined to such a flat layer makes it the datum par excellence to define the galactic pole.

Millman: What is the relative accuracy of the determination of the galactic plane from $21-\mathrm{cm}$ observations and from stars?

Pawsey: This question will be reported on by Commission $33 \mathrm{~b}$ in Moscow. Broadly, the optical observations give a scatter in the derived pole of a few degrees, depending on the type of object selected. The radio observations give a scatter of the order of 0.1 degrees or less. The optical scatter is 
probably associated with the fact that the observations are restricted to a region of about 3500 parsecs radius round the sun and in this region the radio observations indicate large-scale distortions of the galactic disk.

Hoyle: Does the thickness of the sheet of gas remain small compared with its deviation from the mean plane in the outer parts of the galaxy?

Oort: Yes, it does.

Margaret Burbidge: I should like to ask Dr. Oort whether he has any ideas as regards the origin of the circulation of material that comes in from the halo and then moves out from the center into the disk.

Oort: It seems to me that the only known mechanism that could cause the arms in the central part to move away from the center would be magnetic forces. It is important to remember that the arms not only move away from the center, but while doing so they seem to pick up the required angular momentum to keep them rotating.

Burbidge: The presence of the source at the center suggests that the magnetic field may be higher there than in the disk in general.

Edmondson: It is well known that the positions of the radio spiral arms are very sensitive to the model used to compute distances from velocities. A model with systematic inward motions increases the inward spiraling of the radio arms, and a model with outward motions would do the reverse. If the expansion found in the inner part of the Galaxy extends to the outer part, even in reduced amount, this would change the computed positions of the outer arms and make them spiral out in agreement with Lindblad's ideas about spiral structure.

Gold: The outward streaming of gas could result from the redistribution of angular momentum by forces, possibly magnetic, that tended to force the system toward rigid body rotation. Such a tendency would result in an inward motion to the inside of a certain radius, and an outward motion to the outside. This is so for all cases where the gravitational orbits correspond to an angular velocity that diminishes from the center outward. Another point I would like to make concerns the sense in which gas dynamical forces, i.e. pressure or magnetic forces, must be acting on the gas. It is commonly seen in the other spiral systems that the bright blue stars lie mainly on the outside of the densest dust lanes of the spiral arms. This would be the case, as Andrew Young at Harvard has pointed out recently, if these stars had formed recently out of gas in the spiral arms that rotated faster than the gravitational orbit speed. The gas dynamical forces must there be directed inward.

Oort: There is some evidence that at least in the sun's surroundings the gas is not moving away from the center with a very appreciable speed. For if it did, the solar motion derived from moderately old stars could not agree so well with that derived from the gas or from OB stars. This also indicates that magnetic pressure is not likely to be very. important at this distance from the center. In connection with the possibility that magnetic pressure might be the cause of differences between the distribution of the stars and 
the gas, I wish to point out that such differences will occur, whatever mechanism we make responsible for the conservation of spiral structure. It would appear that gas transport between arms is a more promising means for this conservation.

Savedoff: Is there reason to doubt that the plane of the gas is identical to the plane defined by the gravitational field of the predominant stellar masses in the Galaxy?

Oort: I agree that in my answer to Professor Becker I have been overcautious. Personally I would be quite surprised if there was a difference between the plane of the gas and the plane of symmetry of the total mass in the galactic system. I should like to ask a question concerning the theoretical interpretation of the flatness of the gas disk. Does not this indicate that in the past there must have been considerable transport of gas from the inner to the outer parts of the disk?

Mills: I would like to show a slide representing the spiral pattern in the southern galactic regions derived from the Leiden rotational model. It is obvious that the picture is very heliocentric, showing that different models are required on each side of the center. Kerr is at present working on a different method of reduction which he hopes will yield both a rotational model and a sensible spiral pattern.

McVittie: It is remarkable that when self-gravitation and/or magnetic fields are taken into account, very thin moving gas clouds can be produced. Dr. Oort has outlined a magnetohydrodynamic problem of a "source" of gas 2.5 kiloparsecs in radius from which the gas streams out in such a thin cloud. Has this theoretical problem yet been tackled by anyone?

van de Hulst: It has only very recently been established that the expanding motion is a general phenomenon in the central region and not a peculiarity of one spiral arm. No serious theoretical work has been done on this yet. 